\title{
New aspects in Peanut growing technology by Foliar application of Lactofol "Fe" with decreased pesticide doses
}

\author{
N. Shaban ${ }^{1 *}$, E. Kadhum ${ }^{* *}$, E. Loginova ${ }^{* * *}$ and S. Bistrichanov ${ }^{*}$ \\ "University of Forestry, Faculty of Agronomy \\ ${ }^{* *}$ Ministry of Agriculture and Food - Bulgaria \\ ${ }^{* * *}$ IZK "Maritza" - Plovdiv, Bulgaria
}

Rec. 22 May, Accept. 20 July 2010

\begin{abstract}
The mixed application of suspension leaf fertilizer lactofol [lac. Fe] with pesticides [herbicides \& insecticides] stimulated high control effects, which didn't decrease the weed \& insect control affects by reducing the pesticide doses with $12.5 \& 25 \%$. The insecticide control was effective against mites when the lac. Fe was used alone without any mitecides. The yield was increased (7.4-28.4\%) after treatment with mixed application of lac. Fe and pesticides. The highest yield was obtained (28.4\%) when the lac. Fe was mixed with $25 \%$ decrease in pesticide doses. These results certified the economic \& ecological value of this technology.
\end{abstract}

Key words: Peanut, Leaf fertilizer lactofol, Pests, pesticides.

\section{Introduction}

The applications of suspensions of leaf fertilizer lactofolR with pesticides played an important role in sustainable agriculture [Pavlova 1992]. Using of this element in agriculture in same time insure- leaf fertilizing and success weed and insect control in tomato, potato, French bean \& other crops was reported [Velev, 1993, Tailah 1995].The positive effect of fertilizer lactofol can be explained by decreasing the flowing of pesticides from the leaf surface, increasing the velocity of their penetration in leaf stomata, and stimulating vigorous plants. In this work, the effects of applying of lactofolR (alone and in combinations with pesticides) on weeds and mites control as well as the yield of peanut were investigated.

\section{Material and Methods}

This study was achieved, during the period between 1994-1996, at the Experimental Research Station- of Vegetable Growing Department in the Agriculture University Plovdiv Bulgaria. The study determined the changes in the weed vegetation, weed control and the yield of cv. Peanut Calina. The experiment was carried out as described in Table 1 . Conventional hand sprayer was used to apply the different treatments. Weeds were investigated through as follow: annual -number per $\mathrm{m} 2$, and their fresh weight were scaled per g per $\mathrm{m} 2$. Mite effects were investigated through calculating the average number of moving juvenile, adults and mite eggs per 10 leaves. After harvesting, the yield in variants was measured and calculated per da.

\footnotetext{
* Corresponding author:

Dr. N. Shaban

nidalshaban@abv.bg
} 


\begin{tabular}{|c|c|c|c|c|c|c|c|}
\hline \multirow[t]{2}{*}{ Variant } & \multicolumn{3}{|c|}{$I^{\text {st }}$ treatment phase 3-5 true leaf } & \multicolumn{2}{|c|}{$\mathrm{II}^{\mathrm{en}}$ treatment in flowering } & \multicolumn{2}{|c|}{$\begin{array}{l}\text { III }^{\text {th }} \text { treatment } \\
\text { fruiting }\end{array}$} \\
\hline & lact. Fe & Basagran & Fuzilade & lact. Fe & Karate & lact. Fe & Talastar \\
\hline 1 & & & & contrc & & & \\
\hline 2 & 600 & & & 600 & & & 600 \\
\hline 3 & & 300 & +200 & & 30 & & 30 \\
\hline 4 & 600 & +200 & +150 & 600 & +20 & 600 & +20 \\
\hline 5 & 600 & +250 & +175 & 600 & +25 & 600 & +25 \\
\hline 6 & 600 & +300 & +200 & 600 & +30 & 600 & +30 \\
\hline
\end{tabular}

Table (1) The scheme of the treatments (combinations and doses in ml per 1001 water)

\section{Results and Discussion}

The mixed application of lac. Fe with herbicides Basagran \& Fuzilade showed a positive weed control affects against the annual weeds and Sorghum halepenses. The weed control effects varied from 74.4- 83.4\% and from 78.9- $86.0 \%$ in fresh weight [Table 2]. The data showed that, by mixing application of lac Fe with $25-12.5 \%$ decrease of the pesticide doses or with optimal acaricide doses (var. 4, 5, 6) the weed control was very clear. The mite effect can be carried out by treating the plant with lac. Fe in combined with Talastar 10-EK. [var. 2] .The yield of peanut increased during the experiment period. The highest yield was registered in plants from var.4-[4, $857 \mathrm{t} / \mathrm{h}]$ when the yield of the control plants was $3.78 \mathrm{t} / \mathrm{h}$.

\begin{tabular}{|c|c|c|c|c|c|c|c|c|c|c|}
\hline \multirow[b]{2}{*}{ 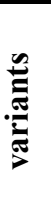 } & \multicolumn{5}{|c|}{ annual weeds - number/5m2 by year } & \multicolumn{5}{|c|}{ fresh weight of weeds- $\mathrm{g} / 5 \mathrm{~m} 2$ by years } \\
\hline & 1994 & 1995 & 1996 & $\begin{array}{c}1994- \\
1996\end{array}$ & $\underbrace{\stackrel{0}{e}}_{\text {: }} e^{0}$ & 1994 & 1995 & 1996 & $\begin{array}{c}1994- \\
1996\end{array}$ & 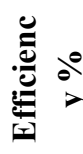 \\
\hline 1. & 114 & 77 & 99 & 96,7 & 0 & 2280 & 1540 & 1680 & 1833 & 0 \\
\hline 2. & 89 & 68 & 13 & 56,7 & 41,4 & 1755 & 1245 & 1310 & 1437 & 21,6 \\
\hline 3. & 26 & 27 & 21 & 24,7 & 74,4 & 429 & 350 & 380 & 386 & 78,9 \\
\hline 4. & 20 & 27 & 5 & 17,3 & 82,1 & 385 & 310 & 160 & 285 & 84,4 \\
\hline 5. & 17 & 24 & 15 & 18,7 & 80,7 & 335 & 225 & 215 & 258 & 85,9 \\
\hline 6. & 16 & 24 & 8 & 16,0 & 83,4 & 315 & 260 & 195 & 257 & 96,0 \\
\hline
\end{tabular}

Table (2) The effect of the treatments with Lactofol Fe and herbicides on the weed's density and their fresh weight (average from three years).

These results confirmed the previous results with other crops (Duncan 1955; Velev, 1993, 1994), and indicated that the mixed application of lactofol with pesticide can be used without any decrease of their effectiveness in spite of decreasing the pesticide doses with 25\% [Velev 1994].
Our data showed that combination of lactofol and pesticides was very promising for applying on the peanut. We could not only realize the effectiveness of control but also the use of fewer amounts of pesticides in combination with lactofol to obtained more yield. These results certify the economical \& ecological value of this technology. 
The highest control effects against weeds and mite Tetanychus urticae was combined with the highest yield of peanuts due to the decreasing the flowing of the using pesticides from the leaf surface, increasing the velocity of their penetration in the leaf stomata and stimulating plant vigorous.

\begin{tabular}{ccccccccc}
\hline & \multicolumn{9}{c}{ Efficiency } & \multicolumn{3}{c}{ after 14days } \\
\cline { 2 - 9 } variants & \multicolumn{9}{c}{ after 7 days } & & \multicolumn{3}{c}{ d } & moving j+a & eggs \\
\cline { 2 - 9 } & $\mathbf{j}$ & 0 & moving j+a & eggs & $\mathbf{j}$ & 0 & 0 & 0 \\
\hline 1. & 0 & 0 & 0 & 0 & 0 & 80,37 & 80,54 \\
\hline 2. & 92,12 & 91,16 & 90,30 & 94,02 & 84,33 & 73,77 & 93,76 & 90,45 \\
\hline 3. & 98,89 & 100 & 99,86 & 99,84 & 95,79 & 82,18 & 53,36 \\
\hline 4. & 99,96 & 99,65 & 99,92 & 99,79 & 89,69 & 37,26 & 80,09 & 75,76 \\
\hline 5. & 99,58 & 96,07 & 99,32 & 98,73 & 92,80 & 54,73 & 89,07 & 62,13 \\
\hline 6. & 99,96 & 99,66 & 99,93 & 99,76 & 82,23 & 69,00 & 76,25 & \\
\hline
\end{tabular}

Table (3) Efficiency of mixes applied of Lactofol Fe and acaricide Talastar 10 EK (calculated by HendersonTilton), Legend: j - juvenile; a - adults;

The mixed application of suspension leaf fertilizer lactofol [lac $\mathrm{Fe}]$ with pesticides [herbicides \& insecticides] stimulate high control effects, which didn't decrease the weed $\&$ insect control effects by reducing the pesticide doses with $12.5 \& 25 \%$. The insecticide control effect

\section{Kg/ dka}

against mites was satisfied, when the lac. Fe as used alone without any mitecides. The results showed us that by the mixed application of lac. Fe \& pesticides, the yield was increase with 7.4$28.4 \%$

(Fig.1).

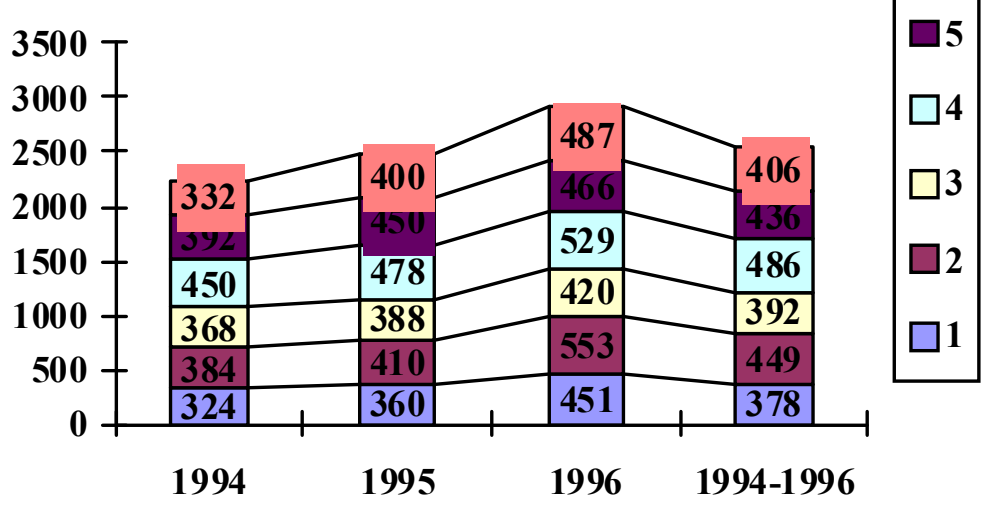

Fig. 1 Peanut yield (nuts) 1994-1996.

The yield increased up to $28.4 \%$ when the lac. Fe was mixed with $25 \%$ decrease in pesticide doses.

\section{References}

Duncan D. 1955. Multiple ranges and multiple tests, Biometrics, II, 1-42.

Pavlova A.P. 1992. Bachvarov, Suspension fertilises Lactofol, Sofia, p.1-29.

Tailah L. 1994. Actual Problems in Eco system Saving"ECO-Ruse-94, 53-56, Ruse, Bulgaria.
Velev B. 1993. Suspensions Lactofol Fertiliser possibilities for decrease of herbicide used together at some vegetable cultures, Agro-eco 1993 Plovdiv, Scientific works vol. 37, 211-214.

Velev B. 1994. Symposium of New tech. in vegetable and Flower production, Ohrid, vol. 1, p. 255-260. 